\title{
OPEN A colonoscopy quality improvement intervention in an endoscopy unit
}

\author{
Rémi Palmier ${ }^{1}$, Thibault Degand ${ }^{1}$, Serge Aho ${ }^{2}$, Côme Lepage ${ }^{1}$, Olivier Facy ${ }^{3}$, \\ Christophe Michiels ${ }^{1}$ \& Sylvain Manfredi ${ }^{1,4} \llbracket$
}

Many studies identified colonoscopy quality indicators in order to improve performance and safety. We conducted a colonoscopy improvement study. Our study was designed according to a Plan-DoStudy-Act cycle: first recording of our quality indicators and identification of shortcomings, second identification of improvement targets and implementation of new procedures, third second recording of quality indicators, fourth validation of procedures and identification of new goals. Quality indicators derived from European and French guidelines were recorded before and after our improvement actions. We were mainly interested in the quality indicators of the colonic preparation, the description of the diagnosed lesions and on the examination reports. The data of 134 patients prospectively included in January-February 2017 were compared to 133 patients included in May-June 2019, after implementation of improvement procedures, in the digestive endoscopy unit of the university hospital of Dijon, France. Our intervention, and in particular the implementation of new standardized forms, improved preparation quality: Boston Bowel Preparation Scale scores increased significantly from 7.8 to 8.2 . Cecal intubation rate increased by $6 \%$, and more adenomas were diagnosed and removed (+3.3\%). Adenoma detection rate increased significantly from 26 to $42 \%$. The completion of withdrawal time measure improved from 6.7 to $100 \%$. Our study led to the rapid implementation of corrective actions and improved quality in our unit and in our personal practice. This quality improvement strategy could be easily implemented in every digestive endoscopy unit.

Colonoscopy is the reference examination for the screening, diagnosis, treatment and surveillance of colorectal diseases. Many recent studies have identified several pre- per- and post-procedure factors related to colonoscopy quality, and these factors have been included in French (French Society of Digestive Endoscopy: SFED) and European guidelines (European Society of Gastrointestinal Endoscopy: ESGE) ${ }^{1-5}$. These quality indicators aim to improve performance and patients' safety. In order to improve colonoscopy quality in the Dijon University Hospital digestive endoscopy unit, we carried out a quality improvement study between January 2017 and June 2019 , in accordance with the latest $\mathrm{ESGE}^{6}$ and $\mathrm{SFED}^{7-9}$ quality indicators. We present our intervention according to the SQUIRE guidelines.

\section{Materials and methods}

The study was a quality improvement initiative and was divided into four phases according to a Plan-Do-StudyAct (PDSA) cycle: first, we recorded our baseline quality indicators, identified shortcomings, established improvement targets and created new tools and procedures; second, we implemented these new tools and procedures; third, we recorded the same quality indicators and compared the results with those of the first period; fourth, we validated the improvement measures and identified new goals.

Baseline record, identification of factors that could be improved and elaboration of new tools and procedures. We included all adult patients referred for a complete screening, diagnostic or therapeutic colonoscopy, with or without general anesthesia at the Dijon University Hospital digestive endoscopy unit between January and February 2017. Patients referred for sigmoidoscopy or for partial colonoscopy were excluded. All colonoscopies were performed by skilled senior practitioners. The factors we considered imple-

${ }^{1}$ Département d'hépato Gastroentérologie et Endoscopie Digestive, Centre Hospitalier Universitaire de Dijon, Dijon, France. 'Département d'hygiène Hospitalière, Centre Hospitalier Universitaire de Dijon, Dijon, France. ${ }^{3}$ Département de Chirurgie Digestive et Oncologique, Centre Hospitalier Universitaire de Dijon, Dijon, France. ${ }^{4}$ Hepato-Gastroenterology Unit, University Hospital, Dijon, France. ${ }^{\circledR}$ email: sylvain.manfredi@chu-dijon.fr 
menting for our quality improvement project were the quality indicators of the European and French colonoscopy quality guidelines. In accordance with the 2017 ESGE and 2018 SFED guidelines ${ }^{6-10}$, we separated quality indicators into three groups: "pre-procedure", "per-procedure" and "post-procedure". We added several quality indicators considered important in our practice: tolerance to the bowel cleansing, evaluated using a questionnaire: Very good/Good/Medium/Bad/Very bad; time to colonoscopy for all patients according to the indication: in particular times between the consultation with the primary care physician, the gastroenterologist, the anesthesiologist, the Fecal immunologic test (FIT) result and the colonoscopy. Time to colonoscopy did not appear as a quality indicator in reference studies, but we considered it a major point, especially for screened patients with a positive fecal immunologic test. The SFED strongly recommends a maximum of 31 days between the FIT gastroenterology visit and the colonoscopy, followed by a printed day-0 mail. The quality indicators and objectives are summarized (Appendix):

After analyzing phase 1 results, we identified weaknesses and set improvement targets. We also created new tools and procedures in accordance with national validated recommendations. The main deficiency identified was the quality of the colonic preparation. The interventions implemented were targeted on the harmonization of prescriptions and protocols for colonic preparation, the collection of the quality of the preparation and its evaluation.

Second phase: implementation of improvement measures. First, we created electronic standardized bowel preparation protocols with simplified order forms available for all practitioners of Dijon University Hospital. These were based on last ESGE, SFED and French society of anesthesiology (SFAR) recommendations ${ }^{9,11-15}$ : all common preparation protocols were available, but we encouraged the use of PEG (Polyethylene glycol) 2 $\mathrm{L}+$ ascorbic acid. All protocols provided split dosing and if possible, end of take between 3 and $5 \mathrm{~h}$ before the colonoscopy.

Second, we created an electronic standardized endoscopic report based on the most recent SFED recommendations $s^{7,8}$, which replaced our old free-text examination report. The main indicators we focused on were withdrawal time, BBPS and polyp characterization. The histology order form was automatically generated according to the information provided in the colonoscopy report. Our aim was also to generate an automatic, printed, standardized colonoscopy report with a day-0 mail for outpatients. In parallel, practitioners were encouraged to train themselves in the use of quality indicators to improve polyp detection and macroscopic analysis. The new documents and procedures were drawn up and developed with all the unit's endoscopists and implemented at the end of 2018 .

Third phase: second recording of quality indicators. We conducted a final evaluation of our practices in May and June 2019, using the same quality indicators. We compared the results before and after the intervention to identify the impact on the different quality indicators.

Fourth phase: validation of improvement measures and identification of new goals. Statistical methods. Continuous variables were expressed as means with standard deviations except for time variables which were expressed as medians with interquartile ranges (IQR) and categorical variables as percentages. Comparison between baseline and final evaluation were performed using Pearson Chi-square test or Fischer exact tests, when appropriate, for qualitative variables, and using Wilcoxon or Kruskal-Wallis tests for quantitative variables. We collected data prospectively and statistics were validated by the statistics unit of our University Hospital. Statistics were done with STATA ${ }^{\circ}$ and SAS $9.4^{\circ}$ software. p-values and p-trends were considered statistically significant based on the 2 -sided probability of 0.05 .

Ethical consideration. In accordance with French law, only oral consent of the participants is necessary and has been obtained.

\section{Results}

Population characteristics. One hundred and thirty-four patients referred for complete colonoscopy were included in the baseline phase between January and February 2017. One hundred and thirty-three patients were included with the same criteria in the final phase between May and June 2019. Patients' characteristics are resumed in Table 1. Patients of phase 1 and 2 were comparable for age, sex and body mass index (BMI). Most patients were outpatients $66.4 \%$ and $68.5 \%$ respectively. Patients' origins in phase 1 were comparable to those in phase 2 .

Pre-colonoscopy indicators. All patients in the study had a dedicated specialist visit before the colonoscopy and a clear indication was available for all colonoscopies. Colonoscopy indications in phase 1 were significantly different from those in phase 2 with respectively more colonoscopies after a positive FIT: $22 \%$ and 4.5\%, fewer colonoscopies for personal history: $8 \%$ and $20 \%$ and fewer colonoscopies for other indications: $8 \%$ and $17 \%(p=0.02)$. The proportion of colonoscopies for symptoms (anemia, abdominal pain, hemorrhage and diarrhea) was similar in the two phases: $38 \%$ and $36 \%$ (Table 1 ). All patients provided consent, and all endoscopy checklists and endoscope disinfections were validated for the two phases.

At the baseline measurement step, a multitude of non-standardized preparation protocols were used by gastroenterologists and no standardized colonoscopy preparation order form was available. We identified this element as an indicator to be improved and we created and implemented an electronic standardized colonoscopy preparation order set with various alternatives, but favoring PEG + sodium picosulfate and split dosing. 


\begin{tabular}{|c|c|c|c|c|c|}
\hline & \multicolumn{2}{|l|}{ Phase 1} & \multicolumn{2}{|l|}{ Phase 2} & \multirow[b]{2}{*}{$P$} \\
\hline & $n=134$ & $(\%)$ & $\mathrm{n}=133$ & $(\%)$ & \\
\hline Men & 73 & 54.5 & 66 & 49.6 & \multirow{2}{*}{0.43} \\
\hline Women & 61 & 45.5 & 67 & 50.4 & \\
\hline \multicolumn{6}{|l|}{ Unit } \\
\hline Outpatients & 89 & 66.4 & 91 & 68.5 & \multirow{3}{*}{0.06} \\
\hline GE inpatients & 22 & 16.3 & 32 & 24.0 & \\
\hline Non GE inpatients & 23 & 17.3 & 10 & 7.5 & \\
\hline \multicolumn{6}{|l|}{ Applicant practitionner } \\
\hline General practitioner & 40 & 29.9 & 57 & 42.9 & \multirow{4}{*}{0.06} \\
\hline Own gastroenterologist & 53 & 39.5 & 37 & 27.8 & \\
\hline Other gastroenterologist & 1 & 0.7 & 3 & 2.2 & \\
\hline Other specialist & 40 & 29.9 & 36 & 27.1 & \\
\hline \multicolumn{6}{|l|}{ Colonoscopy indication } \\
\hline FIT & 30 & 22.4 & 6 & 4.5 & \multirow{6}{*}{0.02} \\
\hline Symptoms & 52 & 38.8 & 48 & 36.1 & \\
\hline IBD & 15 & 11.2 & 17 & 12.8 & \\
\hline Personal history & 11 & 8.2 & 26 & 19.6 & \\
\hline Familial antecedent & 15 & 11.2 & 13 & 9.8 & \\
\hline \multirow[t]{2}{*}{ Other (before surgery) } & 11 & 8.2 & 23 & 17.2 & \\
\hline & Mean & SD & Mean & SD & $p$ \\
\hline Age (years) & 59.9 & 15.4 & 57.9 & 14.5 & 0.17 \\
\hline BMI $\left(\mathrm{kg} / \mathrm{m}^{2}\right)$ & 26.6 & 6.2 & 26.2 & 6.6 & 0.8 \\
\hline
\end{tabular}

Table 1. Population characteristics. FIT: Fecal immunological test, IBD: inflammatory bowel disease, BMI: body mass index, SD: standard deviation, GE: gastroenterology.

This standardized electronic order set included diet procedures for the 3 days before the colonoscopy. In phase 1, the most frequent preparations used were PEG + ascorbic acid in 68 (50.7\%), PEG only in 36 (26.9\%) and sodium picosulfate in 25 patients $(18.6 \%)$. The results were significantly different after implementation of the standardized bowel preparation protocols with an increase in PEG + ascorbic acid, used in 115 patients (86.5\%), and a decrease in both PEG alone, used only in two patients (1.5\%), and sodium picosulfate, used in 11 patients (8.3\%) ( $p=0.0001)$. Split dosing significantly increased from 27 to $97 \%(p=0.0001)$, and the last take between 3 to $5 \mathrm{~h}$ before the procedure increased from 25 to $95 \%(p=0.0001)$. The acceptability level questionnaire was proposed to more than $50 \%$, and almost all of these patients (90\%) reported tolerance to the preparation as good or very good in both phases.

The results for bowel preparations and time to colonoscopy or visit intervals are presented in Table 2.

The median time between the gastroenterologist consultation and the colonoscopy was 38 days (IQR 2-52) in phase 1 versus 53 days (IQR 35-64) in phase $2(p=0.0001)$. In the FIT population $(n=30)$, the median time between the fecal immunologic test result and the colonoscopy was 69 days (IQR 58-97) in phase 1 versus 108 days (median, IQR 103-136) in phase 2 , with a significant difference $(p=0.02)$.

Per-colonoscopy indicators. At the 1st phase, the BBPS was not systematically entered in the colonoscopy report and left free to the choice of the endoscopist. We implemented the requirement to fill in this item in our standardized report, resulting in a BBPS completion increase from 84 to $99 \%(p=0.0001)$ (Table 3). BBPS score distribution is shown in Fig. 1. Obstruction was not considered failure and was excluded from the BBPS analysis. There were five obstructions diagnosed in phase 1 ( 3 cancers and 2 diverticulosis) and three in phase 2 ( 1 cancer and 2 diverticulosis). BBPS greater than 5 (ESGE cut off) increased from 93 to $97 \%(p=0.002)$, and BBPS greater than 6 (SFED cut off) from 80 to $88 \%(p=0.002)$. The average BBPS was significantly better in phase $2(8.2)$ than in phase 1 (7.8) $(p=0.007)$. There was no difference in BBPS between morning and afternoon colonoscopies with a score of 8.11 versus 7.76 , respectively $(p=0.8)$. BBPS was similar whatever the time between the end of the take and the colonoscopy time: less than $5 \mathrm{~h}$ or more than $5 \mathrm{~h}(p=0.23)$. The best preparation results were obtained with PEG + ascorbic acid, PEG alone and sodium picosulfate with average BBPS at 8.2, 7.4 and 6.9, respectively $(p=0.0004)$. For these three preparations, split dosing was associated with an increase in the BBPS $(p=0.03)$ (Table 3). The cecal intubation rate increased non-significantly from 84 to $90 \%(p=0.15)$ (Table 3). Colonoscopy failed in 16 patients in phase 1, four for technical reasons and 12 for insufficient preparation. In phase 2, 10 colonoscopies failed, six for technical reasons and four for insufficient preparation.

Of the 134 colonoscopies performed in phase 1, 44 (32.8\%) were normal; polyps were found in 50 cases (37.1\%), cancer in seven cases (5.2\%), diverticulosis in 15 cases (11.2\%), IBD in eight cases (6\%) and another diagnosis in 10 cases (7.5\%) (2 angiodysplasia, 2 infectious colitis, 2 post-radiation colitis, 1 ischemic colitis and 3 unclassified colitis). Of the 133 colonoscopies performed in phase 2, 50 (37.6\%) were normal, 54 (40.6\%) 


\begin{tabular}{|c|c|c|c|c|c|}
\hline & \multicolumn{2}{|l|}{ Phase 1} & \multicolumn{2}{|l|}{ Phase 2} & \multirow[b]{2}{*}{$p$} \\
\hline & $\mathrm{N}=134$ & $(\%)$ & $N=133$ & $(\%)$ & \\
\hline \multicolumn{6}{|l|}{ Preparation } \\
\hline PEG $4 \mathrm{~L}$ & 36 & 26.9 & 2 & 1.5 & \multirow{6}{*}{0.0001} \\
\hline PEG $2 \mathrm{~L}+$ ascorbic acid & 68 & 50.7 & 115 & 86.5 & \\
\hline Sodium phosphate drinkable & 3 & 2.2 & 0 & 0.0 & \\
\hline Sodium phosphate tablets & 1 & 0.8 & 3 & 2.2 & \\
\hline Sodium Picosulfate & 25 & 18.6 & 11 & 8.3 & \\
\hline Enemas & 1 & 0.8 & 2 & 1.5 & \\
\hline \multicolumn{6}{|l|}{ Splitting } \\
\hline None & 24 & 17.9 & 4 & 3 & \multirow{3}{*}{0.0001} \\
\hline 2 takes one day before & 74 & 55.2 & 0 & 0 & \\
\hline Split dosing & 36 & 26.9 & 128 & 97 & \\
\hline \multicolumn{6}{|l|}{ Schedule } \\
\hline Morning & 122 & 91 & 113 & 85 & \multirow{2}{*}{0.13} \\
\hline Afternoon & 12 & 9 & 20 & 15 & \\
\hline End of take & $\mathrm{n}=105$ & & $\mathrm{~N}=131$ & & \\
\hline $3-5 \mathrm{~h}$ & 26 & 24.8 & 125 & 95.4 & \multirow{3}{*}{0.0001} \\
\hline $5-8 \mathrm{~h}$ & 3 & 2.8 & 3 & 2.3 & \\
\hline$>8 \mathrm{~h}$ & 76 & 72.4 & 3 & 2.3 & \\
\hline Acceptability & $\mathrm{n}=70$ & & 69 & & \\
\hline Excellent & 3 & 4.3 & 8 & 11.6 & \multirow{5}{*}{0.9} \\
\hline Good & 59 & 85.3 & 56 & 81.2 & \\
\hline Middle & 5 & 7.1 & 3 & 4.3 & \\
\hline Bad & 3 & 4.3 & 2 & 2.9 & \\
\hline Very bad & 0 & 0.0 & 0 & 0.0 & \\
\hline \multirow[b]{2}{*}{ Times (days) } & \multicolumn{2}{|l|}{$\mathrm{N}=132$} & \multicolumn{2}{|l|}{$\mathrm{N}=133$} & \multirow[b]{2}{*}{$p$} \\
\hline & Median & $(\mathrm{IQR})$ & Median & (IQR) & \\
\hline Addressing practitioner $-\mathrm{GE}$ & 18 & {$[7-31]$} & 36.5 & {$[13-63]$} & 0.0001 \\
\hline GE-anesthesiologist & 22 & {$[9-36]$} & 37 & [21-49] & 0.0003 \\
\hline GE-colonoscopy & 38 & {$[2-52]$} & 53 & {$[35-64]$} & 0.0001 \\
\hline For FIT & $\mathrm{N}=30$ & & $\mathrm{~N}=6$ & & \\
\hline FIT-GE & 33.5 & {$[27-42]$} & 52.5 & {$[40-81]$} & 0.1 \\
\hline Addressing practitioner-GE & 20 & {$[15-28]$} & 35 & {$[28-60]$} & 0.07 \\
\hline GE-Colonoscopy & 36.5 & [26-39] & 52 & {$[32-63]$} & 0.1 \\
\hline FIT-Colonoscopy & 69 & [58-97] & 108 & [103-136] & 0.02 \\
\hline
\end{tabular}

Table 2. Bowel preparations and time to colonoscopy. FIT: Fecal immunological test, GE: gastroenterologist, IQR: interquartile range, PEG: polyethylene glycol.

revealed polyps, one case of cancer ( $0.8 \%)$, IBD in 15 cases (11.3\%), diverticulosis 11 cases (8.3\%) and another diagnosis in two cases (1.5\%) (Table 3 ).

The number of colonoscopies done under general anesthesia was similar in both phases: $93 \%$ and $98 \%$ $(p=0.08)$.

During the 1st phase, the decision to describe the polyps according to the Paris and Kudo classifications was left free to the endoscopist. Following phase 1, we included these items in our electronic report. Completion of the Paris polyp classification was done in only $56.4 \%$ of cases in phase 1 , increasing to $92.7 \%$ of cases in phase 2 $(p=0.0001)$. The Kudo polyp classification was poorly reported in both phases, $9 \%$ and $11 \%$, respectively $(p=0.3)$. The polyp retrieval rate was $100 \%$.

Polyp pathology was significantly different in the two phases; there were more hyperplastic polyps in phase 1: $32.7 \%$ versus $10.9 \%$, and more adenomas in phase $2: 87.3 \%$ versus $54.5 \%(p=0.003)$ (Table 4$)$. The polyp detection rate was not significantly different between phase $1(37 \%)$ and phase $2(41 \%)(p=0.5)$. The adenoma detection rate (calculated for the endoscopy unit) was significantly higher in phase $2(41.7 \%)$ than in phase 1 $(25.8 \%)(p=0.01)$ (Table 4$)$. For FIT indications, the adenoma detection rate was $50 \%$ (30 colonoscopies) in phase 1 versus $83 \%$ in phase 2 with only six colonoscopies $(p=0.12)$.

Post-colonoscopy indicators. Concerning immediate complications in phase 1 , there was no perforation and only one hemorrhage after polypectomy, which was treated during the same colonoscopy, without recurrence. In phase 2, there was one perforation in a context of diverticulosis without polypectomy, and no hemorrhage or inhalation. Information on delivery of the day-0 mail was not easily available for the 1 st phase. 


\begin{tabular}{|c|c|c|c|c|c|}
\hline & \multicolumn{2}{|c|}{ Phase 1} & \multicolumn{2}{|c|}{ Phase 2} & \multirow[b]{2}{*}{$p$} \\
\hline & $\mathbf{n}$ & $(\%)$ & $\mathbf{n}$ & $(\%)$ & \\
\hline BBPS & 125 & & 133 & & \\
\hline Completion in report & 105 & 84 & 123 & 99 & 0.0001 \\
\hline Guidelines objectives & 105 & & 123 & & \\
\hline BBPS $>6$ (SFED) & 98 & 93 & 119 & 97 & 0.002 \\
\hline BBPS > 7 (ESGE) & 84 & 80 & 109 & 88 & \\
\hline \multirow[t]{2}{*}{ Average BBPS } & 105 & & 123 & & \\
\hline & 7.76 & & 8.22 & & 0.007 \\
\hline Colonoscopy results & 134 & & 133 & & \\
\hline Normal & 44 & 32.8 & 50 & 37.6 & 0.02 \\
\hline Polypes & 50 & 37.3 & 54 & 40.6 & \\
\hline Malignancy & 7 & 5.2 & 1 & 0.8 & \\
\hline Diverticulosis & 15 & 11.2 & 11 & 8.3 & \\
\hline Active IBD & 8 & 6 & 15 & 11.3 & \\
\hline Others $^{\star}$ & 10 & 7.5 & 2 & 1.5 & \\
\hline \multicolumn{6}{|l|}{ Cecal intubation } \\
\hline Yes & 113 & 84.3 & 120 & 90.2 & 0.15 \\
\hline No & 21 & 15.7 & 13 & 9.8 & \\
\hline Failure & 16 & 12 & 10 & 7.5 & \\
\hline Technical reasons & 4 & 25 & 6 & 60 & 0.09 \\
\hline Bad preparation & 12 & 75 & 4 & 40 & \\
\hline
\end{tabular}

Table 3. BBPS and colonoscopy results. BBPS: boston bowel preparation scale, SFED: French society of digestive endoscopy, ESGE: European society of gastrointestinal endoscopy, IBD: inflammatory bowel disease. ${ }^{\star} 2$ angiodysplasia, 2 infectious colitis, 2 post-radiation colitis, 1 ischemic colitis and 3 unclassified colitis. Significant values are in bold.

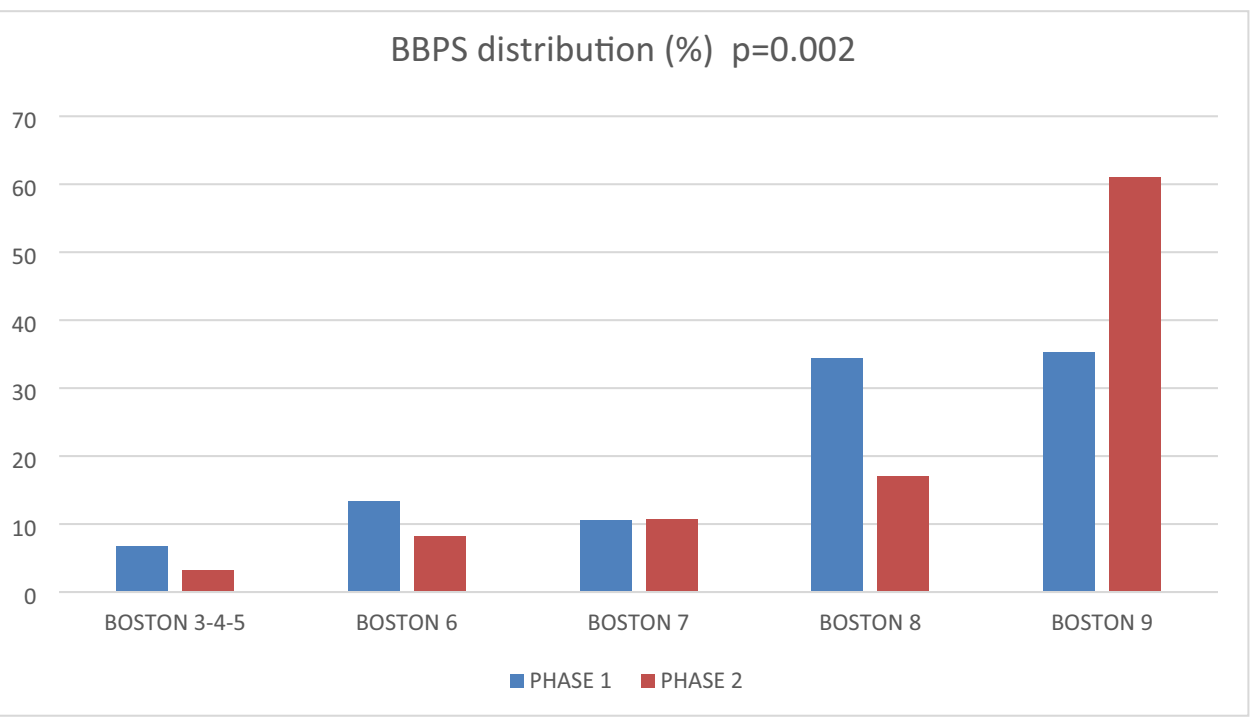

Figure 1. Boston Bowel Preparation Scale distribution.

We included the automatic generation of the letter in our electronic report. For outpatients, a day-0 mail and printed endoscopic report were generated for and delivered to only 24 patients (27\%) during phase 1 versus 91 patients $(100 \%)$ during phase $2(p=0.0001)$.

\section{Discussion}

The main improvements in our practices were the development and standardization of our electronic colon preparation and examination report forms, and the fact that they were made available to all hospital practitioners on the intranet server. This new strategy led to an improvement in the quality of colon preparation for our patients and in the quality of our examination reports, with ESGE and SFED objectives largely achieved. These results are in accordance with previous interventional studies: standardization and implementation of a uniform 


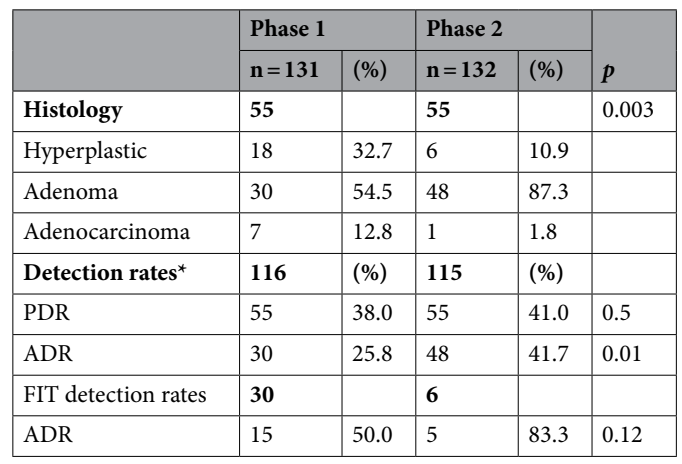

Table 4. Histology and adenoma detection rate. ${ }^{\star}$ After exclusion of patients with IBD and emergency situations ( $\mathrm{n}=15$ in phase 1 and $\mathrm{n}=17$ in phase 2). PDR: Polyp detection rate, ADR: adenoma detection rate. Significant values are in bold.

bowel preparation procedure, and in particular split dosing, increased the proportion of "good" (more than 90\% of mucosa visualized) and "excellent" (more than 95\%) results from $84.6 \%$ to $86.5 \%$ with fewer "fair" results and no change for "poor" results (around $5 \%)^{16}$. In a cohort study of inpatients, the benefit of a colonoscopy preparation electronic order form favoring split dosing was shown, with $86 \%$ of good preparation versus $43 \%$ before the intervention ${ }^{17}$. Another study also showed the benefit of an electronic order form, as the proportion of colonoscopies with inadequate preparation was significantly reduced and the diagnostic yield modestly improved ${ }^{18}$. In addition, the use of an electronic order form increased the mention of BBPS scores in colonoscopy reports from 0.5 to $85 \%{ }^{17}$ and favorably impacted compliance with follow-up guidelines in an average-risk screened population ${ }^{19}$. The implementation of an electronic colonoscopy preparation order set had the strongest impact in an interventional study ${ }^{20}$ that also included the education of physicians and patients. The overall result was a reduction in the rate of poor colonoscopy preparations from 19 to $4 \%$.

The inclusion of a polyp description paragraph in the standardized endoscopy report probably explains the decrease in the proportion of hyperplastic polyp resections and the increased proportion of adenoma resections. The Paris classification is the main polyp classification used in our electronic endoscopic reports, even though the Kudo classification does not give the same information (crypt structure and so histology prediction). The preference can be explained by the simplicity of the Paris compared to the Kudo classification, which needs more time for precise examinations and electronic color enhancement. The Kudo classification was used only for large polyps and only by one practitioner, but should be used every time to predict polyp histology. In our study, practitioners provided predictions of polyp histology, but did not clearly use the Kudo classification in endoscopic reports.

ESGE and SFED recommend an ADR greater than 25\% for diagnostic colonoscopies (IBD, planned polyp resections and emergencies excluded) and greater than $45 \%$ for FIT indications. In our study, even if this rate was not individual, this objective was reached in both phases, and also improved in phase 2 . This increase in the ADR and PDR could probably be related to the improvement in the quality of colon preparation, in particular the use of split dosing, as shown by Seo et al. ${ }^{21}$. For FIT colonoscopy indications, the ADR cannot be considered because of the small number of colonoscopies performed, due to a temporary interruption of the mass screening program in France at the time of the study.

The withdrawal time item was included in the standardized colonoscopy report and was longer than 6 min (ESGE major criterion) in all cases.

Our new electronic colonoscopy report made it possible to generate a day- 0 mail and a printed report, which was delivered to all outpatients when they go back home.

Time to the colonoscopy sadly increased between the two phases of our study. This could be explained by the development in our unit of advanced therapeutic colonoscopy techniques with more complex polyp resections. It is necessary to increase the number of endoscopy procedures under general anesthesia to reduce times to endoscopy access. The SFED strongly recommends a maximum of 31 days between an FIT gastroenterology visit and the colonoscopy ${ }^{7,8}$. We were close to this objective during the first phase of the study but we did not have enough patients with a positive FIT to show significant results. Proposals to reduce the time between specialist consultations and the colonoscopy would require dedicated consultation schedules and increased access to operating rooms (OR), but currently all OR slots are already saturated and the margin to increase their number is narrow.

The strengths of this study are the choice of internationally validated quality indicators with recent, clear recommendations. We also studied other non-validated but interesting indicators, such as times between the consultation with a gastroenterologist or anesthesiologist and the colonoscopy, to take stock of our practices and follow their evolution over time.

Nevertheless, there are several limitations in this work. First, the two populations were not exactly comparable in term of colonoscopy indications. This is simply explained by the smaller number of Fecal Immunologic Tests in France at the time of the second phase. This was a single-center study, our conclusions therefore cannot be generalized to all endoscopy centers. Each center has its own procedures, and these procedures must be assessed in each center to identify factors that could be improved before implementing specific procedures. Another limitation is the variability among physicians in rating the preparation. This could be a source of bias, even 
though we used the standardized, recognized BBPS. For the future, there is still room for progress, especially in colonoscopies with a BBPS score of 7 or more, and in cecal intubation rates, which should rise to $90-95 \%$. Concerning adenoma detection rates, a greater number of patients are needed to make a robust analysis and obtain significant results. Withdrawal time is at least $6 \mathrm{~min}$, but should tend towards $10 \mathrm{~min}$, and the precise measurement of this time is needed.

\section{Conclusion}

Our study allowed us to identify shortcomings in our procedures, and as a result to rapidly implement new corrective actions to increase the quality of our unit and our personal practices, particularly in the quality of colon cleansing, which led to an increase in the adenoma detection rate. In addition, our intervention resulted in an increase in the completion rates for several items, such as BBPS and the Paris classification, and the automatic generation of a day-0 mail and printed colonoscopy report. Our intervention required an initial analysis of the shortcomings of our unit and can therefore only be generalized to units that have identified the same shortcomings. However we think that this program is feasible and easy to implement, and results in the improved quality, safety and performance of a digestive endoscopy unit. Our study could serve as an example to encourage administrators of endoscopy units to undertake creative and effective actions to improve colonoscopy quality. This could easily be done in every digestive endoscopy unit.

Quality improvement is a steady, continuous effort, and we need to continue auditing and improving to get closer to what is best for our patients.

Received: 11 June 2021; Accepted: 13 December 2021

Published online: 17 January 2022

\section{References}

1. Rembacken, B. et al. Quality in screening colonoscopy: Position statement of the European Society of Gastrointestinal Endoscopy (ESGE). Endoscopy 44, 957-968 (2012).

2. Rizk, M. K. et al. Quality indicators common to all GI endoscopic procedures. Gastrointest. Endosc. 81, 3-16 (2015).

3. Hadlock, S. D. et al. The quality of colonoscopy reporting in usual practice: Are endoscopists reporting key data elements?. Can. J. Gastroenterol. Hepatol. 2016, 1-7 (2016).

4. Imler, T. D. \& Imperiale, T. F. Measuring the quality of colonoscopy: Where are we now and where are we going?. Gastrointest. Endosc. 82, 520-522 (2015).

5. Rees, C. J. et al. Expert opinions and scientific evidence for colonoscopy key performance indicators. Gut 65, 2045-2060 (2016).

6. Kaminski, M. F. et al. Performance measures for lower gastrointestinal endoscopy: A European Society of Gastrointestinal Endoscopy (ESGE) quality improvement initiative. United Eur. Gastroenterol. J. 5, 309-334 (2017).

7. Lapuelle J, Bernardini D, Robaszkiewicz M, Chaussade S. Critères de qualité de la coloscopie: SFED 2018. https://www.sfed.org/ files/files/coloscopie_critqual.pdf.

8. Bernardini D, Lapuelle J, Chaussade S, Robaszkiewicz M. Critères de qualité du compte rendu de coloscopie : SFED 2018. https:// www.sfed.org/files/files/coloscopie_cptrend_critqual.pdf.

9. Conseils d'administration de la Sfed et de la Sfar, Bulois P, Bazin JE, Lapuelle J, Al-Nasser B, Chaussade S, et al. Préparation colique et anesthésie générale: position commune SFED/SFAR. Acta Endosc. 2017; 47: 2-5.

10. Lai, E. J., Calderwood, A. H., Doros, G., Fix, O. K. \& Jacobson, B. C. The Boston Bowel Preparation Scale: A valid and reliable instrument for colonoscopy-oriented research. Gastrointest. Endosc. 69, 620-625 (2009).

11. Parente, F., Marino, B. \& Crosta, C. Bowel preparation before colonoscopy in the era of mass screening for colo-rectal cancer: A practical approach. Dig. Liver Dis. 41, 87-95 (2009).

12. Kilgore, T. W. et al. Bowel preparation with split-dose polyethylene glycol before colonoscopy: A meta-analysis of randomized controlled trials. Gastrointest. Endosc. 73, 1240-1245 (2011).

13. Hassan, C. et al. Bowel preparation for colonoscopy: European Society of Gastrointestinal Endoscopy (ESGE) Guideline. Endoscopy 45, 142-155 (2013).

14. Bucci, C. et al. Optimal bowel cleansing for colonoscopy: Split the dose! A series of meta-analyses of controlled studies. Gastrointest. Endosc. 80, 566-576.e2 (2014).

15. Martel, M. et al. Split-dose preparations are superior to day-before bowel cleansing regimens: A meta-analysis. Gastroenterology 149, 79-88 (2015).

16. Menees, S. B., Kim, H. M. \& Schoenfeld, P. Split-dose bowel preparation improves adequacy of bowel preparation and gastroenterologists' adherence to National Colorectal Cancer Screening and Surveillance Guidelines. World J. Gastroenterol. 24, 716-724 (2018).

17. Yadlapati, R. et al. An automated inpatient split-dose bowel preparation system improves colonoscopy quality and reduces repeat procedures. J. Clin. Gastroenterol. 52, 709-714 (2018).

18. Sullivan, B., Zhang, C., Wegermann, K., Lee, T. H. \& Leiman, D. A. Standardizing inpatient colonoscopy preparations improves quality and provider satisfaction. Int. J. Health Care Qual. Assur. 33, 277-287. https://doi.org/10.1108/IJHCQA-11-2019-0186 (2020).

19. Menees, S. B. et al. The impact of bowel cleansing on follow-up recommendations in average-risk patients with a normal colonoscopy. Am. J. Gastroenterol. 109, 148-154 (2014).

20. Shah-Khan, S. M., Cumberledge, J. \& Reynolds, G. J. Using the plan-do-study-act approach to improve inpatient colonoscopy preparation. BMJ Open Qual. 6, e000230 (2017).

21. Seo, J. Y. et al. Multidirectional colonoscopy quality improvement increases adenoma detection rate: Results of the Seoul national university hospital healthcare system Gangnam center colonoscopy quality upgrade project (Gangnam-CUP). Dig. Dis. Sci. 65, $1806-1815$ (2020).

\section{Author contributions}

R.P. and S.M. designed the research study and wrote the paper. R.P., S.M., T.D., C.M., C.L. performed the research and collected data. S.A. analysed the data. O.F. reviewed the paper. All authors reviewed and approved the final version of the article, including the authorship list. 


\section{Competing interests}

The authors declare no competing interests.

\section{Additional information}

Supplementary Information The online version contains supplementary material available at https://doi.org/ 10.1038/s41598-022-04786-y.

Correspondence and requests for materials should be addressed to S.M.

Reprints and permissions information is available at www.nature.com/reprints.

Publisher's note Springer Nature remains neutral with regard to jurisdictional claims in published maps and institutional affiliations.

(c) (1) Open Access This article is licensed under a Creative Commons Attribution 4.0 International License, which permits use, sharing, adaptation, distribution and reproduction in any medium or format, as long as you give appropriate credit to the original author(s) and the source, provide a link to the Creative Commons licence, and indicate if changes were made. The images or other third party material in this article are included in the article's Creative Commons licence, unless indicated otherwise in a credit line to the material. If material is not included in the article's Creative Commons licence and your intended use is not permitted by statutory regulation or exceeds the permitted use, you will need to obtain permission directly from the copyright holder. To view a copy of this licence, visit http://creativecommons.org/licenses/by/4.0/.

(c) The Author(s) 2022 